\title{
Stato filosofico nel paziente in trattamento dialitico
}

\author{
Sandro Scipioni ${ }^{1}$, Lucia Giuliani ${ }^{2}$, Valentina Micheluzzi ${ }^{3}$, Francesco Burrai ${ }^{4}$ \\ ${ }^{1}$ Dipartimento di Nefrologia e Dialisi, Asur Marche Area Vasta 3, Macerata - Italia \\ ${ }^{2}$ Dipartimento Asur Marche Area Vasta 3, Macerata - Italia \\ ${ }^{3}$ UC Cardiologia e UTIC, ASSL Sassari, Sassari - Italia \\ ${ }^{4}$ Formazione e Ricerca, ATS Sardegna, Sassari - Italia
}

\begin{abstract}
Philosophical state in a dialysis treatment patient
The following study is original respect to the scientific panorama in fact, through a literature review that uses a qualitative metasynthesis method and follows the aggregative or meta-aggregative approach of the Joanna Briggs Institute, it returns to the reader 3 main results: the Anguish for an imminent death, the relationship with the body, sexuality, pain and dimension of the person, and the Perception of time and existentialism. Reflecting on the "philosophical state or philosophy of the patient in dialysis treatment" through these 3 macro-themes means investigating in depth the words of the patients, extrapolating their feelings, fears and worries, so as to understand their overall view of life and illness even in the most veiled aspects. Finally, the reflection will be used to direct the care of professionals towards a real and meaningful appointment of the dialysis patient. All thanks to the philosophy that goes beyond the methodology of research and evaluation of data, through the interpretation, direction and demonstration of the concept of limit, as an intrinsic part of man, disease and science.
\end{abstract}

Keywords: Existentialism, Narrative, Philosophy, Psychology

\section{Introduzione}

Partiamo da un'opera di Pablo Picasso, Scienza e carità (1897). Ci troviamo in una stanza grigia e lugubre, nella quale una donna dal volto angosciato è distesa su un letto che occupa la maggior parte del dipinto: alla sua destra un medico le misura i battiti cardiaci, alla sua sinistra una suora la accudisce. La centralità del dolore dell'ammalata si colloca così tra scienza e carità, due elementi che da un lato sembrano in opposizione, ma che, dall'altro, sembrano completarsi e collaborare.

Rifacendoci all'etimologia dei termini, la parola "scienza" deriva dal verbo latino "scire", che significa sapere; in particolare le scienze mediche si occupano di conoscere e studiare il corpo umano al fine di curare i pazienti.

Received: December 02, 2019

Accepted: December 31, 2019

Published online: February 20, 2020

Indirizzo per la corrispondenza:

Francesco Burrai

Formazione e Ricerca, ATS Sardegna

Sassari- Italia

francesco.burrai@libero.it
Il termine "carità", invece, prende la sua origine dal latino "caritas", che significa benevolenza, amore e compassione, che, a sua volta, deriva dal latino "cum patior", soffro con, cioè un sentimento di una persona che è in grado di percepire la sofferenza dell'altro desiderando di alleviarla. Il concetto di compassione richiama quello di empatia, che indicava il rapporto emozionale di partecipazione soggettiva che legava lo spettatore del teatro greco antico all'attore recitante e anche una tecnica di recitazione per l'immedesimazione dell'attore con il personaggio che interpretava. Nelle scienze umane l'empatia rappresenta un atteggiamento verso gli altri caratterizzato dalla volontà di comprenderli mettendo da parte il più possibile i sentimenti e i giudizi personali.

Alla luce di queste definizioni, la domanda che risulta sospesa nell'atmosfera malinconica del quadro è: può la scienza, da sola, salvare l'uomo o è altrettanto importante curare lo spirito e l'anima?

Indagare sullo "stato filosofico o della filosofia nel paziente in trattamento dialitico" significa riflettere sull'essere umano affetto da patologia renale e significa indagare sul senso dell'essere e dell'esistenza umana, tentando di definire i limiti della conoscenza, della natura e delle sue possibilità.

Indagare sul paziente in trattamento dialitico comporta, inoltre, individuare i caratteri di maggiore sofferenza per tracciare un percorso in cui inserire un processo di accoglienza e cura.

L'importanza dello studio che presentiamo va rintracciata, quindi, non solo nella volontà di rispondere a domande tanto 
esistenziali quanto fondamentali per l'uomo affetto da patologia renale, ma anche nella mancanza di ricerche adeguate.

\section{Metodologia}

Attraverso una breve revisione di letteratura nelle principali banche dati, Pubmed e Cinahl comprese, vediamo come nessun risultato possa essere estrapolato in toto per essere incluso nella ricerca. La scarsità degli elementi per un'adeguata analisi del caso ci suggerisce come la medicina non abbia ancora preso molto in considerazione l'aspetto filosofico del paziente.

In ogni modo, la ricerca effettuata nelle banche dati ha permesso di rintracciare un quadro all'interno del quale catturare i tratti che contraddistinguono principalmente lo stato filosofico del paziente dialitico.

La review usa il metodo della metasintesi qualitativa e segue l'approccio aggregativo o meta-aggregativo del Joanna Briggs Institute (1). Sono stati inclusi solo studi primari condotti con ogni tipo di metodologia qualitativa senza esclusione di tempo, provenienza o lingua.

I risultati ottenuti dai motori di ricerca sono stati letti, analizzati e inclusi nello studio da 2 ricercatori indipendenti. Una prima cernita è stata effettuata leggendo titolo e abstract, quindi leggendo l'intero articolo. Gli articoli individuati da ciascun ricercatore sono stati nuovamente discussi per ottenere un accordo sugli articoli da selezionare.

Sebbene gli articoli estratti non possano risultare sufficienti per una revisione sistematica della letteratura, lo studio che segue vuole indirizzare verso una comprensione filosofica del paziente dializzato, quindi verso un'analisi qualitativa attraverso le parole estrapolate dagli articoli e dagli studi fenomenologici/ermeneutici intrapresi fino a oggi (2).

\section{Risultati}

I risultati possono dividersi in 3 macrotemi principali: "Angoscia per una morte imminente", "Il rapporto con il corpo: sessualità, dolore e dimensione della persona", "Percezione del tempo ed esistenzialismo".

\section{Angoscia per una morte imminente}

La malattia cronica implica nel concetto stesso un'idea limitata di tempo e di vita. II paziente "lives with limitations and with a think about death" (3). II passaggio tra una seduta dialitica e l'altra è breve, talmente breve da preoccupare il paziente rispetto a un futuro incerto, vicino e segnato dai tanti trattamenti invadenti. La strategia per poter affrontare con più serenità l'angoscia di una morte incombente è il rifugio nel presente e nella propria famiglia: "close to the family" è l'espressione utilizzata per sottolineare il rapporto stretto, arroccato in modalità difensiva con gli affetti della famiglia. Anche quella ricerca bramata del rifugio è un concetto filosofico: "Catone Uticense si volse a Platone solo quando ciò che per lui era l'ordine supremo delle cose, la libertà della sua Repubblica, fu distrutto; allora cercò un rifugio in un ordine trascendente. Ma quando il corso naturale delle cose creò nel seno di quegli Stati liberi un'aristocrazia, a cui poco per volta il popolo abbandonò la cura della cosa pubblica, e dal cozzo di queste aristocrazie si levò l'ordinamento burocratico e militare della servitù romana, allora la via fu aperta al cristianesimo" (4). È su questo spazio, del rifugio, che si muovono storicamente anche l'arte, la religione ed ecco la filosofia.

La consapevolezza di una morte imminente genera paura e il senso dell'imminenza della morte è costante nei pensieri dei pazienti intervistati, come se ogni atto medico ne sollecitasse il pensiero (5): "Patients voiced with certainty that they knew they would die, but when and how this would occur was uncertain" (6).

"The last time when I had cardiac arrest, I was out for 50 minutes! ... The doctors declared me dead... and isn't it amazing that they brought me back after 50 minutes, and I still have a brain and that my brain wasn't cooked?" (6).

I pazienti quindi sperimentano direttamente $o$ indirettamente episodi acuti come ictus, embolia, infarto e così via, che contribuiscono a creare la sensazione di morte imminente e di precarietà della vita.

"Several factors contributed to a general sense of feeling unsafe, such as having problems with catheters and fistulas, feeling frightened by episodes of infiltration that caused swelling in the arms, and learning of patients who died" (7).

"I have heard [about] a lot of people that died on dialysis and had strokes on dialysis... Once I sit down there, I don't know whether I'm gonna come out alive or dead" (8).

\section{II rapporto con il corpo: sessualità, dolore e dimensio- ne della persona}

Essere subordinato a un corpo malato è frustrante (9).

Il corpo, indebolito dalle sedute dialitiche e dalla malattia, ha bisogno di mantenimento (dieta, idratazione, fisioterapia, massaggi, cure e controlli), poiché detta un ritmo diverso: si altera nella frequenza cardiaca, negli atti respiratori e nei movimenti muscolari e scheletrici. La persona è spazialmente connessa con la dimensione del suo corpo che occupa un posto nuovo e predominante rispetto alla persona in salute. In fondo, lo spazio non è mai concepibile come una realtà a sé, fuori dai corpi, e Aristotele ci ricorda che è il luogo, per definizione, sempre "luogo-di-qualcosa" ed è come la superficie che delimita un corpo (10). Il luogo dell'uomo è il suo corpo, che è simile a un recipiente. Di questo recipiente si conoscono i bordi e la capacità di accogliere la malattia, "il limite che l'uomo sperimenta nel corpo non si deve imputare alla sua natura o sostanza, bensì alla sua corruzione" (11).

"I worry constantly... Is it [body] going to be able to keep up and keep going?" (5). Non è da sottovalutare, nel cambiamento del ritmo del corpo, anche l'aspetto sessuale. La sessualità è un elemento chiave per calcolare la percezione di 
qualità di vita del paziente (12). Infatti, la sessualità fa parte di ogni singola persona umana ed è naturalmente oggetto di modificazione durante l'arco della vita, nella quale subisce cambiamenti ciclici o meglio ritmici (13). L'inserimento brusco di una malattia nella sessualità può comportare disturbi psicologici severi importanti, tanto quanto la malattia è impattante (atto, percezione, piacere, condivisione, paura).

La sessualità riguarda il corpo e il desiderio è uno stato del corpo: spesso la presenza del catetere può rappresentare per molti pazienti e per il loro partner un problema psicologico di cambiamento della propria immagine. Malgrado ciò, attraverso un processo di accettazione del catetere e grazie al supporto del partner, il sesso e l'intimità possono continuare a essere un piacere. Anche per alcuni fattori dell'impotenza possono esserci motivi corporali: vascolari, neurologici, ormonali, farmacologici e psicologici. Le persone affette da insufficienza renale cronica possono presentare alcune complicanze legate alla malattia e al trattamento e tra queste ricordiamo l'aterosclerosi (una patologia degenerativa delle arterie), che può compromettere la circolazione a livello genitale causando una disfunzione erettile. Infine, anche lo stress della malattia e del trattamento dialitico può incidere sull'aspetto psicologico, causando preoccupazione, apprensione, timore e quindi uno stato di ansia che può influire negativamente sul desiderio sessuale.

Rivolgendo ancora una volta lo sguardo alla filosofia per chiedere aiuto, arriviamo al desiderio sessuale di Sartre, Terza parte di L'Essere e II Nulla, in cui l'autore presenta "il secondo atteggiamento nei confronti dell'altro". "Bisogna rinunciare completamente all'idea che il desiderio sia desiderio di voluttà o desiderio di far cessare un dolore" e Sartre ritiene adeguato il poter definire il Desiderio per mezzo del suo oggetto trascendente, quell'oggetto che va inevitabilmente incontro alla propria soppressione proprio perché oggetto di desiderio. Se all'Altro è dedicato l'oggetto del Desiderio, è Ricoeur a confutare con l'opera "Sé come un altro" (2) la tesi, oltre che a rappresentare il moto oscillatorio (ritmico) perenne costitutivo dell'identità soggettiva. L'autore intende porre l'enfasi sulla dimensione del "tra" (il sé e l'alterità), inteso come spazio di giuntura e condizione stessa del processo del riconoscimento. II "tra" è anche lo spazio della "dissimmetria originaria", attraverso la quale si manifestano l'estraneità (interna ed esterna al soggetto) e la "distanza" incolmabile e costitutiva del rapporto del sé con l'altro. Il corpo ne è il mezzo, lo strumento.

Infine, il ritmo che viene a mancare è quello della quotidianità, "The second experience of space-time embraced in our analysis, rhythm, is the embodied experience of repetition and difference in everyday life (14)", che ci riconduce inevitabilmente all'ultimo tema, il tempo.

\section{Percezione del tempo ed esistenzialismo}

La temporalità è il senso ontologico della nostra inquietudine (15).
"Hovering between living in the present and worrying about the future: The participants tried to focus on living in the present. This emerged as one way to handle the heavy burden of knowing that they would undergo dialysis treatment for the rest of their lives. Moreover, thinking of the future meant thinking of worries and sometimes difficult decisions. Their futures were already behind them, and so it was difficult to think ahead, as facing the future meant facing death. "I don't want to think that far. I just want 1 week at a time to pass, and cope with dialysis//there are several [fellowpatients] who have died over the years, and I don't want to think about things like that. I want to concentrate on having as good time as possible" (14).

La temporalità scandisce l'esistere nel presente e nella malattia, nel proiettarsi in maniera distorta verso un futuro incerto.

Da sempre la percezione del tempo alterata dalle malattie ha suscitato interesse in ambito bio-medico perché indice di stress, qualità della vita mutata e malessere (16). Rispondere alle questioni della percezione del tempo nel paziente in trattamento dialitico significa quindi approcciare un bisogno fondamentale del paziente e prendersi cura della malattia in senso pieno. Dare maggior valore alla speranza e all'affettività significa inoltre riempire alcune lacune lasciate dalla sensazione del tempo che si sta perdendo.

\section{Conclusioni}

La dialisi è una forma di terapia che ha importanti ripercussioni emotive sul paziente a causa dei numerosi tipi di stress a cui il paziente è sottoposto. L'infermiere è la figura professionale che per prima e con maggiore attenzione risponde a questi bisogni, inoltre è colui che trascorre più tempo con il malato, trovandosi quindi nella posizione più favorevole per esprimere al paziente un'assistenza non solo tecnicomateriale, ma anche psicologico-esistenziale.

Nell'aspetto psicologico del paziente gli elementi che abbiamo analizzato in questa review sono:

1) I'angoscia per una morte imminente; 2) il rapporto con il corpo: sessualità, dolore e dimensione della persona; 3) percezione del tempo ed esistenzialismo, che mutano nel paziente in trattamento dialitico e che, indagati in modo critico, offrono elementi per la costituzione di una rete assistenziale infermieristica efficace.

Se il concetto di "Cura" ha subito nel corso della storia profonde modificazioni, indicava una figura allegorica e mitologica nell'antichità greco-romana, mentre, in epoca tardo anticamedievale, era già diffuso nel contesto teologico e, successivamente, nell'epoca moderna, il concetto acquisiva uno spazio narrativo: il prendersi cura rimane un atteggiamento strutturalmente relazionale, che porta l'io fuori dal sé, dall'autoreferenzialità all'eteroreferenzialità. Così, "la cura è il filo rosso di una riflessione etico-antropologica nonché ontologica, che mostra l'identità nascosta dell'uomo e della relazione (17). 


\section{Disclosures}

Financial support: No financial support. Conflict of interest: No conflict of interest.

\section{Bibliografia}

1. The Joanna Briggs Institute. Joanna Briggs Institute Reviewers' Manual. Adelaide: JBI. 2014.

2. Ricoeur P. Interpretation Theory: Discourse and the Surplus of Meaning. Forth Worth: Christian University Press. 1976.

3. Cesarino GB, Casagrande LDR. Paciente com insuficiência renal crônica em tratamento hemodialítico: atividade educativa do enfermeiro. Revista latino-americana de enfermagem. 1998; 6(4):31-40.

4. Martinetti P. Saggi e discorsi. Torino: Paravia. 1926.

5. Cervantes L, Jones J, Zoucha J, Fischer S. Experiences and Values of Latinos with End Stage Renal Disease: A Systematic Review of Qualitative Studies. Nephrol Nurs J. 2016;43(6): 479-93.

6. Calvin AO, Engebretson JC, Sardual SA. Understanding of advance care planning by family members of persons undergoing hemodialysis. West J Nurs Res. 2014;36(10):1357-73.

7. Ivie JM. Developing an in-house resource for clinical social work services in the dialysis setting: a pilot program. Adv Chronic Kidney Dis. 2006;13(3):295-306.
8. Tijerina MS. Mexican American women's adherence to hemodialysis treatment: a social constructivist perspective. Soc Work. 2009;54(3):232-42.

9. Axelsson L, Randers I, Jacobson SH, Klang B. Living with haemodialysis when nearing end of life. Scand J Caring Sci. 2012;26(1):45-52.

10. Ruggiu L. Fisica, Aristotele. Milano: Mimesis. 2017.

11. Alici L. La città di Dio, Sant'Agostino. Milano: Bompiani. 2001.

12. Santos PR, Capote JR Jr, Cavalcanti JU, et al. Quality of life among women with sexual dysfunction undergoing hemodialysis: a cross-sectional observational study. Health Qual Life Outcomes. 2012;10:103.

13. Freud S. Opere. Vol. 4: Tre saggi sulla teoria sessuale e altri scritti (1900-1905). Torino: Bollati Boringhieri. 1905.

14. McQuoid J, Jowsey T, Talaulikar G. Contextualising renal patient routines: Everyday space-time contexts, health service access, and wellbeing. Soc Sci Med. 2017;183:142-50.

15. Paci E. Esistenzialismo e Storicismo. Milano: Mondadori. 1950.

16. Giuliani L, Ghilardi G. La percezione del tempo nei pazienti di cure palliative: una revisione della letteratura. Metodologia Didattica e Innovazione Clinica. 2014;22(1):66-70.

17. Giuliani L. La cura nel tempo, Tempus fugit. Milano: Mimesis. 2018. 